\title{
Device- and Analytics-Agnostic Infrastructure for Continuous Inpatient Monitoring: A Technical Note
}

\author{
Noé Brasier ${ }^{a, d}$ Lukas Geissmann ${ }^{c}$ Miro Käch ${ }^{c}$ Markus Mutke ${ }^{a, b}$ \\ Bianca Hoelz $^{a}$ Fiorangelo De leso ${ }^{a, b}$ Jens Eckstein ${ }^{a, b}$ \\ ${ }^{a}$ CMIO Research Group, D\&ICT Department, University Hospital Basel, Basel, Switzerland; \\ ${ }^{b}$ Department of Internal Medicine, University Hospital Basel, Basel, Switzerland; ${ }^{C}$ Leitwert ${ }^{\circledR}$ \\ $\mathrm{GmbH}$, Zürich, Switzerland; dDepartment of Internal Medicine and Emergencies, \\ Kantonsspital Obwalden, Sarnen, Switzerland
}

\section{Keywords}

Wearable sensors · Analytics · Monitoring · Digital biomarkers

\section{Abstract}

The internet of healthcare things aims at connecting biosensors, clinical information systems and electronic health dossiers. The resulting data expands traditionally available diagnostics with digital biomarkers. In this technical note, we report the implementation and pilot operation of a device- and analytics-agnostic automated monitoring platform for in-house patients at hospitals. Any available sensor, as well as any analytics tool can be integrated if the application programming interface is made available. The platform consists of a network of Bluetooth gateways communicating via the hospital's secure Wi-Fi network, a server application (Device Hub) and associated databases. Already existing access points or low-cost hardware can be used to run the gateway software. The platform can be extended to a remote patient monitoring solution to close the gap between in-house treatments and follow-up patient monitoring.

(C) 2020 The Author(s)

Published by S. Karger AG, Basel

\section{Introduction}

Monitoring in-house patients is labour intense and often delayed in documentation and interpretation. Therefore, assessing vital signs is limited during the day. The internet of healthcare things connects biosensors with clinical information systems to enable automated continuous patient monitoring [1]. Recorded sensor data can be stored, shared and analysed to provide longitudinal insights in health and disease [2, 3]. 
Table 1. Comparison of different platforms in 5 categories

\begin{tabular}{llllll}
\hline $\begin{array}{l}\text { Platform } \\
\text { comparison }\end{array}$ & $\begin{array}{l}\text { Device } \\
\text { agnostic }\end{array}$ & $\begin{array}{l}\text { Data on } \\
\text { premise }\end{array}$ & $\begin{array}{l}\text { Dedicated } \\
\text { gateways }\end{array}$ & $\begin{array}{l}\text { Inpatient } \\
\text { monitoring }\end{array}$ & $\begin{array}{l}\text { For clinical } \\
\text { research }\end{array}$ \\
\hline Device Hub & $\checkmark$ & $\checkmark$ & $\checkmark$ & $\checkmark$ & $\checkmark$ \\
RADAR-CNS & $\checkmark$ & $\checkmark$ & $\times$ & $\times$ & $\checkmark$ \\
Actigraph & $\times$ & $\times$ & $\checkmark$ & $\times$ & $\checkmark$ \\
BioBeat & $\times$ & $\times$ & $\checkmark$ & $\checkmark$ & $\times$ \\
\hline
\end{tabular}

Device agnostic means it is possible to integrate any device with accessible API from different manufacturers; data on premise means that data can be stored on premise providing full data sovereignty to the hospital; dedicated gateways means it includes dedicated gateway solution for passive data collection from wearables (i.e., without smartphones); inpatient monitoring means the platform is targeting wearable-based vital sign monitoring of inpatients and aims to be embedded into hospital care routines; for clinical research means it provides a solution for clinical studies with wearable devices. The specific platforms were chosen as a representative for larger groups of similar platforms. The Leitwert Device Hub is a novel device agnostic and open infrastructure presented in this paper. $R A D A R-C N S$ is a research platform integrating wearables via smartphones. Actigraph is a commercial end-to-end solution where a company offers the device including connectivity and data cloud. BioBeat is a closed and proprietary system for patient monitoring where the company offers the device including connectivity and data cloud.

Emerging biosensors are part of an end-to-end solution including cloud-servers, mobile applications and proprietary algorithms (Fig. 1a). Most communicate via Bluetooth due to low-power requirements and require a gateway to connect to a webserver. For consumer devices, the gateway function is usually performed by a mobile phone. However, the use of multiple end-to-end systems with separate user interfaces without full transparency on applied algorithms or data sovereignty is not practicable in hospitals. A Bluetooth network to integrate devices is not part of the typical hospital infrastructure yet.

We report a novel device- and analytics-agnostic automated platform to continuously record digital biomarkers of in-house patients using a network of Bluetooth gateways (Fig. 1b; Table 1). Various devices can be integrated and operated on the same system if the application programming interface (API) is accessible. Raw and processed data is streamed and stored in a secure on-premise data warehouse. Data can be subsequently or continuously analysed by third-party analytics tools. This infrastructure can be extended to remote patient monitoring, which closes the gap between in-house and follow-up patient monitoring after hospital discharge. While some of the components are certified medical products, the end-toend system has not yet been certified as a medical product.

\section{Connectable Smart Biosensors}

Connected biosensors are providing a patient-driven, non-invasive and continuous way to collect digital biomarkers. Despite the high number of available sensors, only few wearables are sufficiently clinically validated for healthcare applications. This motivates the implementation of the reported infrastructure, which enables hospitals to validate such applications independently. Highly modular biosensors can be combined to collect context driven information consisting of various digital biomarkers. This flexibility allows to match the varying demands of heterogeneous patients. 
Digital

Biomarkers a

End-to-end solution


$$
\stackrel{\uparrow}{\stackrel{1}{ล}}
$$

b

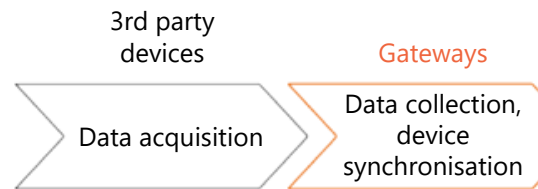

(A)

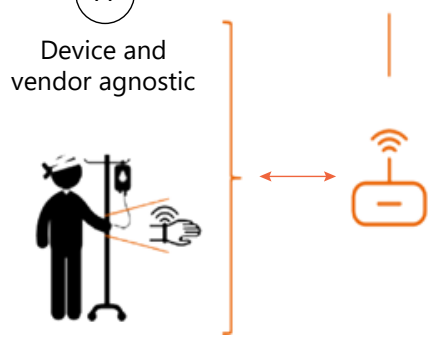

c



Hospital



(D)

(C)
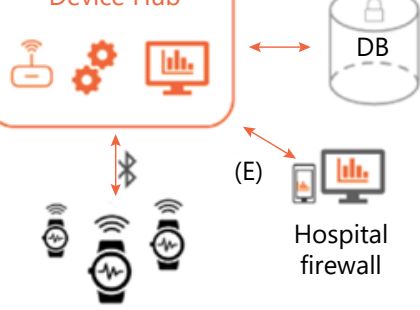

Server

Data, device

and patient management

(B)

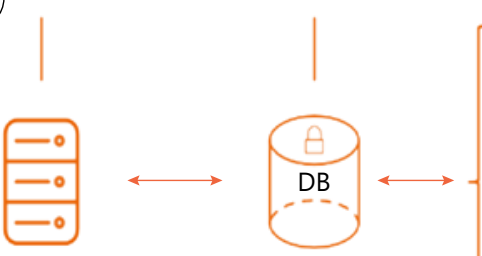

(C)

Analytics,

processing,

visualisation

م0\% 
Examples of biosensors are:

Accelerometer sensors. Sensors record the acceleration of the on-body device itself along the 3 axes of a Cartesian coordinate system. Inertial measurement units are embedded into novel sensor models and allow to measure three-dimensional movement. The devices are worn on the upper and, lower extremity, can be fixed on the thorax or plugged into the ear.

Photoplethysmographic (PPG) sensors. PPG sensors record changes of light absorption in skin translumination of blood vessels. Changes are due to intraluminal, pulsatile differences and primarily provide feedback from heartrate [4].

Further sensors. A broad range of novel devices such as sweat and breath sensors will enable a next generation, non-invasive molecular diagnostics by extending the standard of care $[3,5]$.

Once a biosensor has been selected, it can easily be integrated into the platform ecosystem.

\section{Data Streaming}

The platform consists of a network of Bluetooth gateways communicating via the secure hospitals Wi-Fi network with the server application (Device Hub) and associated databases (Fig. 1b).

The gateway software can be packaged in a docker container to deploy it on existing access points inside the hospital or dedicated low-cost hardware. It collects high-resolution time-series data from Bluetooth devices which it forwards via a Wi-Fi or mobile network to the Device Hub. Direct device-to-server communication using Wi-Fi or mobile networks is also possible. The connection between a Bluetooth device and the gateway is established automatically as soon as the patient wearing the device gets within reach of a gateway. If multiple gateways are available, the Device Hub dictates, which gateway establishes the connection. By broadly deploying gateways, patients move freely while data is synchronised continuously between the wearable device and the Device Hub. If no gateway is within reach data can be buffered temporarily on the device.

The encryption between devices and gateways depends on the capabilities of the device. The reported system supports TLS for HTTP and MQTT with end-to-end encryption between devices and the Device Hub or point-to-point encryption from device to gateway and gateway to Device Hub. Devices can authenticate themselves towards the server based on pre-shared secrets or one-time authentication performed during the integration of a new device, ensuring that only trusted devices can connect to the server.

Fig. 1. A novel device- and analytics-agnostic infrastructure for automated inpatient monitoring using wearable biosensors for data acquisition. a End-to-end solution vs. hospital. In typical consumer-focused end-toend solutions, the smartphone (A) provides 3 key functionalities: (1) managing the identity of the wearer; (2) orchestrating data collection and communication with server and device; and (3) sometimes pre-processing and visualising the data. The data is further processed and stored in the device manufacturer's cloud (B). As smartphones are not a practicable gateway solution for stationary hospitals the Device Hub (C) provides this missing functionality at the hospital. The data can be stored on hospitals premise (D) while also allowing authorised personnel to access the data (E). Additionally, it is possible to integrate third-party analytics tools via API (F), creating an ecosystem for data analytics services while preserving the privacy of patients' data. b The linear monitoring chain and its modules. A novel device- and analytics-agnostic infrastructure for automated patient monitoring using wearable biosensors for data acquisition (A) based on a network of gateways, servers and databases for device- and data management, user workflows and data storage (B) and open interfaces to use third-party analytics for automated data processing and visualisation (C). c A patient's vital parameter stream from the pilot trial iMove. Monitoring accelerometer data and vital parameters. A, accelerometer data (3-axis Cardesien coordinate) over 15 min; B, heart rate (HR) matched to A; 1, patient advised to sit down calm on a chair for $30 \mathrm{~s}$; 2, patient advised to walk around; bpm, beats per minute.

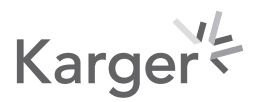


The gateway software is fully integrated with the Device Hub, allowing system administrators to monitor the uptime of the gateway network in real time, to change gateway settings and keep track of the connections established with devices.

The core of the system is the Device Hub, a webserver application built on NGINX and leveraging the open-source framework Django. It can be deployed in the premise of a hospital on a virtual machine, is hardened against common cyber-attacks, avoids logic errors based on custom code validation and ensures consistent code based on domain specific language. It provides standard processes to define roles and permissions, to record an audit-log of all interactions, to manage device settings and updates, for device-to-patient assignment and revocation, to monitor incoming data streams and to perform data transformations. The data recorded is made available to third-party applications via an open REST API with TLS support or can be processed on the server itself, for example, to enrich data streams with metadata like the ID of the currently assigned patient. It allows building application specific workflows, for example, for clinical trials or early warning scores, and forms the basis for the systematic integration of manufacturers-independent wearable devices and analytics into clinical processes.

The architecture of the system allows to store data either on cloud servers or on local hospital databases, transferring the data sovereignty to the healthcare provider and offering the required flexibility to comply with local data protection regulations.

\section{Analytics, Processing and Visualisation}

Learning algorithms and artificial intelligence allow to process large amounts of data. In the Device Hub, incoming data streams are automatically and continuously analysed, interpreted and aggregated with metadata. The open interfaces of the reported infrastructure allow the integration with analytics applications from third-party providers, creating an ecosystem for data applications. This is necessary to meet the many different requirements in a complex healthcare environment such as a University Hospital. Increasing numbers of novel analytical applications are available that may be integrated in the reported infrastructure such as:

Automated screening for atrial fibrillation (aFib). There are validated algorithms available to automatically detect atrial fibrillation, which is associated with a significant higher risk for stroke [6]. These algorithms use either electrocardiography or PPG signals, and analyse a potential beat-to-beat irregularity [4, 7].

Automated gaitmeasurement. Surgery can have a significant impact on a patient's mobility [8]. Accelerometer devices easily and continuously provide data about a patient's movement patterns. Maintaining prehospitalisation community mobility is associated with increased risk of death, nursing home admission, and functional decline $[9,10]$. Monitoring physical activation may further help to prevent deconditioning and pressure ulcers.

\section{Implementation Costs}

The University Hospital Basel and Leitwert ${ }^{\circledR} \mathrm{GmbH}$ started a development partnership. Initial investments to setup the pilot installation amount to CHF 60,000 and were realised within 3 months. In-kind contributions of the University Hospital included consultations on specifications and specific requirements on a regular basis, whereas Leitwert ${ }^{\circledR} \mathrm{GmbH}$ contributed the already existing server platform and extended it with relevant functionality. The Department of Digitalization and ICT of the University Hospital Basel supported the project by providing server infrastructure and supporting the integration of the access points into the secure Hospital network. Hardware costs were limited to Raspberry Pi computers,

\section{Karger'}


which are used as gateways ( $<$ CHF 100/piece including SD cards, casing and power supply). The mobile sensors were a loan free of charge from Biovotion ${ }^{\circledR}$ (regular price approximately CHF 600/piece). For implementation, 4 months' 0.2 FTE from each the CMIO research group and Leitwert ${ }^{\circledR} \mathrm{GmbH}$ were needed and remain as fixed costs for maintenance.

\section{Clinical Testing and Application}

The reported infrastructure has been implemented in an observational trial at the University Hospital Basel. This project aims to develop a monitoring system based on wearable medical devices that allows a continuous vital monitoring of activity and heart rate and will allow to gain insights into activity level and health as well as indicate if patients at risk of fall are getting up from bed (Fig. 1c). So far, data is accessed after recording without any quality warning system, which is intended to be integrated at a later stage.

\section{Outlook}

Automated, continuous and synchronous recordings of vital signs combined with diagnostic algorithms will provide deeper insights in a patient's physiology and disease. This dynamic and modular approach has a great potential contributing to a more patient-centred therapy and being cost-effective and easy to implement in hospitals despite the various requirements of medical specialities. An early detection of deterioration may further improve clinical outcome and thereby potentially reduce healthcare costs. Nevertheless, challenges during the initial project implementation such as the laborious linking of anonymised data to a patients' folder, the limited amount of sensor buffering time when out of gateway reach and the novelty of device handling need to be addressed during the further developing process.

\section{Statement of Ethics}

The iMove study was approved by the Ethics Committee of north-western Switzerland (EKNZ) and patients provided informed consent.

\section{Conflict of Interest Statement}

M.K. is co-founder of Leitwert ${ }^{\circledR}$ and has an equity interest in the company. L.G. is staff member of Leitwert ${ }^{\circledR}$. Leitwert ${ }^{\circledR}$ has a commercial interest in the developed infrastructure and may potentially benefit from the research results. J.E., M.M., and F.D.I. are staff members of the University Hospital of Basel. N.B. was a staff member of the University Hospital of Basel from 2017 to 2019 and was a staff member of Leitwert ${ }^{\odot}$ during an internship in 2019. J.E. is holding $0.5 \%$ of virtual shares of Preventicus ${ }^{\odot}$.

\section{Funding Sources}

This infrastructure project was granted by the University Hospital Basel, Switzerland. Leitwert ${ }^{\circledR}$ is a business and development partner of the University Hospital of Basel, Switzerland.

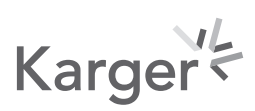




\section{Author Contributions}

J.E. and M.K. contributed substantially to the conception of the infrastructure. N.B., J.E., and M.K. critically interpreted state-of-the art literature. N.B., J.E., M.K., L.G., and F.D.I. drafted the work and finally approved the version to be published. N.B. and J.E. agreed to be accountable for all aspects of the work in ensuring that questions related to the accuracy or integrity of any part of the work are appropriately investigated and resolved. M.M., B.H. revised the manuscript, approved the final version and were significantly involved into the development of the project as well as conducting the iMove study.

\section{References}

1 Firouzi F, Rahmani AM, Mankodiya K, Badaroglu M, Merrett GV, Wong P, et al. Internet-of-Things and big data for smarter healthcare: from device to architecture, applications and analytics. Future Gener Comput Syst. 2018;78:583-6.

2 Babrak LM, Menetski J, Rebhan M, Nisato G, Zinggeler M, Brasier N, et al. Traditional and Digital Biomarkers: Two Worlds Apart? Digit Biomark. 2019 Aug;3(2):92-102.

3 Brasier N, Eckstein J. Sweat as a Source of Next-Generation Digital Biomarkers. Digit Biomark. 2019 Dec;3(3): 155-65.

4 Brasier N, Raichle CJ, Dorr M, Becke A, Nohturfft V, Weber S, et al. Detection of atrial fibrillation with a smartphone camera: first prospective, international, two-centre, clinical validation study (DETECT AF PRO). Europace. 2019 Jan;21(1):41-7.

5 Hashoul D, Haick H. Sensors for detecting pulmonary diseases from exhaled breath. Eur Respir Rev. 2019 Jun; 28(152):190011.

6 Wolf PA, Abbott RD, Kannel WB. Atrial fibrillation as an independent risk factor for stroke: the Framingham Study. Stroke. 1991 Aug;22(8):983-8.

7 Halcox JP, Wareham K, Cardew A, Gilmore M, Barry JP, Phillips C, et al. Assessment of Remote Heart Rhythm Sampling Using the AliveCor Heart Monitor to Screen for Atrial Fibrillation: The REHEARSE-AF Study. Circulation. 2017;136:1784-94.

8 Berian JR, Mohanty S, Ko CY, Rosenthal RA, Robinson TN. Association of Loss of Independence With Readmission and Death After Discharge in Older Patients After Surgical Procedures. JAMA Surg. 2016; 151(9):e161689.

9 Keppler AM, Nuritidinow T, Mueller A, Hoefling H, Schieker M, Clay I, et al. Validity of accelerometry in step detection and gait speed measurement in orthogeriatric patients. PLoS One. 2019 Aug;14(8):e0221732.

10 Brown CJ, Foley KT, Lowman JD Jr, MacLennan PA, Razjouyan J, Najafi B, et al. Comparison of Posthospitalization Function and Community Mobility in Hospital Mobility Program and Usual Care Patients: A Randomized Clinical Trial. JAMA Intern Med. 2016 Jul;176(7):921-7.

\section{Karger'}

